\title{
Entre el Buen Vivir y el feminismo: avances, desafíos y encrucijadas
}

Feminismo y Buen Vivir. Utopías decoloniales.

VARELA, Soledad; ZARAGOCÍN, Sofía. (Compiladoras).

Cuenca/Ecuador: PYDLOS Ediciones, 2017.

La obra se propone destacar los avances en materia de equidad de género en el Ecuador y en Bolivia, desde la adopción del concepto del Buen Vivir en las Constituciones de 2008 y 2009, respectivamente. Al mismo tiempo, tiene el objetivo de establecer la indisolubilidad de los derechos de las mujeres y de la desigualdad de género con los derechos de los pueblos indígenas y comunitarios, y con las otras desigualdades existentes en estos países.

Si bien es cierto que las Constituciones de ambos países, así como las leyes promulgadas para su aplicación y la redacción de algunas de las políticas públicas formuladas, incorporan de forma vigorosa las necesidades específicas de las mujeres y las relaciones de poder entre los géneros (reales y utópicas), su incorporación no fue una tarea fácil desde el movimiento de mujeres y las mujeres organizadas. Además, aún están pendientes algunos temas que se consideran contradictorios con el concepto del Buen Vivir.

En la obra se muestra como los feminismos que más se acercan al concepto del Buen Vivir son el feminismo comunitario y el feminismo decolonial ${ }^{2}$, pero que también se pueden establecer puentes con otros enfoques feministas, como la economía feminista, para garantizar una verdadera complementariedad armónica entre hombres y mujeres como reclama el Chacha Warmi ${ }^{3}$.

\section{(c) $\odot$}

Esta obra tem licença Creative Commons.

1 También conocido como Vivir Bien, Sumak Kawsay en lengua quichua y Suma Qamaña en lengua aimara. El concepto del Buen Vivir no presenta una autoría individual, sino colectiva, representando una Epistemología del Sur propiamente indígena (ZARAGOCIN, 2017, p. 22).

2 El feminismo decolonial surge en América Latina y prioriza la intersección de género, clase y raza, cuestionando el feminismo universalista occidental; el feminismo comunitario se origina en Bolivia, cuenta con un fuerte componente indígena y se centra en la necesidad de construir comunidad.

${ }^{3}$ El Chacha Warmi (hombre-mujer) es un concepto utilizado en la cosmovisión andina que representa un código de conducta basado en el principio de dualidad y de lo complementario. Se utiliza especialmente para reivindicar la equidad de género, como forma de alcanzar el equilibrio y la armonía de la convivencia humana. 
Bolivia y Ecuador presentan estrategias diferenciadas en lo que respecta a la integración, en el Buen Vivir, del Chacha Warmi y de lo que podríamos llamar la continuidad de las luchas feministas y de mujeres, optando, en el primer caso, por un objetivo de despatriarcalización-descolonización ${ }^{4} y$, en el segundo, por trabajar con la noción misma de equidad de género. En ambos casos se ha percibido un rechazo a los movimientos feministas, pero se ha respetado más el concepto de género, tal vez por ser una categoría relacional que conversa bien con el Chacha Warmi: la mujer existe porque existe el varón y viceversa, y la meta es que la existencia de ambos se complemente, sin relaciones de poder de uno sobre el otro, sino de equilibrio, armonía y complementariedad.

Sin embargo, una verdadera igualdad de género se continúa interpretando como contradictoria con el Buen Vivir, cuando se trata, por ejemplo, de los derechos reproductivos o de la libre orientación sexual. Si bien estas reivindicaciones se interpretan como imposiciones de agendas eurocéntricas, oriundas del feminismo occidental blanco, también es cierto que es difícil asegurar cómo se daban las relaciones entre los géneros antes de la invasión europea. Como indica Gisela Espinosa Damián: "Cierto que, a más de cinco siglos de mestizaje e imposición, es difícil descifrar qué tanto pesa en el sexismo indígena la cultura occidental y qué tanto pesan los elementos culturales mesoamericanos." (Gisela ESPINOSA DAMIÁN, 2014, p. 250). Del mismo modo, Óscar González Gómez (2014) relata como la existencia de los cuilonime entre los mexicas en el siglo XVI, registrados en las crónicas de colonizadores y evangelizadores, nos muestra la posibilidad de otras formas de vivir el sexo, la reproducción social y el género, así como otro tipo de relaciones sociales en la América precolombina.

Las compiladoras de la obra ya nos adelantan en la "Introducción" que "el enfoque del Buen Vivir para este libro es plural, cambiante, anclándose en análisis feministas y con enfoque de género para su devenir conceptual" (Soledad VARELA; Sofía ZARAGOCÍN, 2017, p. 8). De este modo, los diferentes artículos nos muestran los avances realizados en la incorporación de los derechos específicos de las mujeres en las políticas públicas dentro del Buen Vivir, pero también los límites encontrados y los desafíos existentes. Se nos invita a incorporar la igualdad de género en el Buen Vivir, aprovechando de su capacidad cambiante y plural, y su abertura a excluir las discriminaciones e incluir la armonía y el equilibrio humano.

La obra se apoya en el concepto de colonialidad del género desarrollado por María Lugones (2008) y basado a su vez en el concepto de colonialidad de Aníbal Quijano (2000). Además, en ese anclaje con el Buen Vivir, la obra nos muestra cómo el feminismo se aúna a la ecología, entendiendo que la lucha por la igualdad de género debe ir de la mano con la lucha por proteger la Madre Tierra, la Pachamama ${ }^{5}$. Este es un aspecto reivindicado por los feminismos comunitarios.

Este libro representa un esfuerzo en colocar a dialogar el Buen Vivir, como una propuesta decolonial, con teorías feministas y de desarrollo, que en principio se consideran opuestas. La abertura del Buen Vivir, desde su concepción, a dialogar y retroalimentarse con otras formas de vivir, y no como una visión esencialista y aislada, es la mejor puerta de entrada para estos debates, debates importantes y necesarios en esta continua construcción del Buen Vivir en Bolivia y Ecuador. Al mismo tiempo, es una crítica a las limitaciones que están

${ }^{4}$ Uno de los lemas bolivianos de la última década es "No se puede descolonizar sin despatriarcalizar" (basado en el libro de María Galindo, 2013).

${ }^{5}$ Pachamama es una diosa totémica representada por el planeta Tierra, a la que se le hacen ofrendas en las ceremonias agrícolas y ganaderas, rituales provenientes del pueblo Inca que se continúan realizando actualmente en el mundo andino. Es el núcleo del sistema de creencias de actuación ecológico-social entre los pueblos andinos centrales. 
presentes a la hora de aplicar el Buen Vivir en las políticas públicas, más aún cuando se lo quiere aplicar junto con otros enfoques como el de género o interseccionalidad. El énfasis dado a analizar el Buen Vivir en las políticas públicas se explica, en palabras de las propias compiladoras de la obra, por

la persistencia de la exclusión y racialización de las mujeres indígenas que han quedado fuera de las políticas públicas del Estado, a pesar de que éste se apropió de la filosofía del Buen Vivir, la hizo parte de su discurso y propuestas y más adelante dejó de lado a los y las intelectuales que durante décadas construyeron aquella propuesta teórica, moral y revolucionaria llamada Buen Vivir. (VARELA; ZARAGOCÍN, 2017 , p. 10)

La obra cuenta con nueve textos de autoras diferentes, además de la "Introducción" de las compiladoras Soledad Varea y Sofía Zaragozín. Sofía Zaragozín es, también, la autora del primer texto "Feminismo Decolonial y Buen Vivir", donde resalta que, en Ecuador, al contrario de temas como el desarrollo y la interculturalidad, la equidad de género desde los aportes del feminismo académico, activista y político no fue considerada en el concepto del Buen Vivir, pues se consideró el feminismo como una crítica a la modernidad, pero una extensión crítica de la misma, es decir como parte de la propia modernidad. Si bien eso es cierto en el caso del feminismo occidental, no puede aplicarse a los feminismos decoloniales ni comunitarios, como apunta la autora. Zaragozín critica además que ese tema haya sido trabajado en el Buen Vivir por hombres académicos, y reivindica que "la hibridación, el feminismo decolonial es el enfoque feminista más relevante para recalcar la potencialidad utópica y política del Buen Vivir desde el espacio del Abya Yala" (Sofía ZARAGOCín, 2017, p. 18). Si bien Benedict Anderson (2006) nos ilustraba con las comunidades imaginadas, Zaragozín reivindica el Abya Yala' como la "geografía imaginada" (ZARAGOCíN, 2017, p. 19) en la que pueden encontrarse el Buen Vivir y el feminismo decolonial latinoamericano, como un espacio geopolítico e histórico. La autora apuesta por Buenos Vivires en plural, donde se dé una hibridación entre el feminismo decolonial y el feminismo comunitario con el Buen Vivir: "no es entre la modernidad y lo no-moderno, lo colonial y lo no-colonial sino entre procesos comunes y parejos, en proceso de descolonización" (ZARAGOCín, 2017, p. 19).

Aída Hernández en su texto "Confrontando la Utopía Desarrollista: el 'Buen Vivir y la Comunalidad' en las Luchas de las Mujeres Indígenas" llama la atención para recuperar el significado original de las propuestas del Buen Vivir, elaboradas por intelectuales indígenas, pero posteriormente apropiadas por los Estados y despojadas de su potencial crítico, de su carácter revolucionario para romper con el paradigma de la modernidad. Su texto es una crítica amarga a la puesta en práctica de las ideas del Buen Vivir, y en general a las agendas multiculturales, ejecuciones de políticas públicas que no guardan armonía con lo reflejado en las Constituciones de Bolivia y Ecuador. En palabras de la autora:

No obstante los compromisos contraídos en estas nuevas legislaciones, en los últimos años, estamos siendo testigos de un retroceso en el reconocimiento de los derechos políticos y territoriales de los pueblos indígenas y de una embestida de violencia y despojo contra sus territorios y recursos naturales. Los Estados latinoamericanos, incluyendo aquellos que se reconocen como plurinacionales como son Ecuador y Bolivia, vienen imponiendo megaproyectos extractivos mineros, petroleros, forestales; de energía e infraestructura como represas, proyectos eólicos y carreteras; y promoviendo la siembra de monocultivos y transgénicos en los territorios de los pueblos indígenas, sin consulta ni consentimiento previo, libre e informado. En nombre del "progreso y el desarrollo" se está justificando nuevamente el despojo y la violencia en contra de los pueblos originarios. (Rosalva Aída HERNÁNDEZ, 2017, p. 27)

\footnotetext{
${ }^{6}$ Abya Yala es el nombre en la lengua kuna que se refería al territorio ancestral antes de la invasión española en el continente. Si bien no hay pruebas que sustenten la idea de que el término se refiera a la totalidad del continente americano, Abya Yala se ha tomado como geografía imaginada en oposición al nombre dado por los pueblos no originarios, europeos, de América. Es por tanto una posición ideológica desde los pueblos indígenas originarios
} 
Hernández recupera las voces académicas que critican las llamadas reformas multiculturales. Por un lado, se apropian de las agendas, pero al mismo tiempo descentralizan las acciones en las propias comunidades indígenas, no responsabilizándose de las mismas. Según la autora, esto "responde a la necesidad de una agenda neoliberal por descentralizar y promover una sociedad civil más participativa" (HERNÁNDEZ, 2017, p. 27) y se relaciona con el concepto de "multiculturalismo neoliberal" de Charles R. Hale, un multiculturalismo que abre las puertas al diálogo, pero que describe y prescribe qué tipo de indígena y qué tipo de política pueden ser implementadas; donde se establecen los indios permitidos ${ }^{7}$, aquellos que no amenacen el avance de las políticas neoliberales en su conjunto (Charles HALE, 2004, p. 16).

Rosalva Aída Hernández critica igualmente el esencialismo en las luchas indígenas que oculta las relaciones desiguales de género; no reconoce las demandas del movimiento de mujeres indígenas, que generalmente se ven como amenazas al movimiento indígena por considerarse erróneamente demandas individuales frente a demandas colectivas; y relega a las mujeres a su vínculo con la sabiduría popular, colocando al mismo tiempo una responsabilidad de manutención de los saberes populares y las lenguas indígenas que no les competen exclusivamente a ellas. En este sentido, se registra el trabajo realizado por la Red de Mujeres del Chimborazo (Ecuador) para colocar el tema de la violencia de género en el proceso de la Constituyente.

Hernández también critica las posturas del feminismo hegemónico que se oponen con las demandas del movimiento indígena y de las propias mujeres indígenas, posturas que colocan la laicidad por encima de la espiritualidad indígena, y los derechos sexuales y reproductivos por encima de otros derechos que pueden ser considerados de mayor prioridad para las mujeres indígenas. El texto finaliza retomando la propuesta de Boaventura de Sousa Santos (2009) de "ecología de los saberes", donde

una ecología de saberes feministas no desecharía todos los conocimientos acumulados por el feminismo occidental, sino que trataría de relativizar su capacidad heurística, contextualizando sus orígenes y su espacio de enunciación, a la vez que desestabilizaría su relación jerárquica con los conocimientos emancipatorios de las mujeres indígenas, musulmanas, campesinas. (HERNÁNDEZ, 2017, p. 41).

En "La Sostenibilidad de la vida como eje para Otro Mundo Posible", Silvia Vega apuesta por la economía feminista por centrarse en el reconocimiento de la esfera reproductiva, dedicada a sostener la vida humana; o en términos más economicistas, a asegurar la reposición de la fuerza de trabajo. Si en lugar de pensar en términos capitalistas, trabajamos ese reconocimiento de la esfera reproductiva, que recae principalmente en las mujeres, como un vínculo intrínseco con la productiva, en armonía con los recursos de la naturaleza, su integración en el Buen Vivir sólo puede contribuir. De este modo, la autora teje la categoría de la sostenibilidad de la vida en el Buen Vivir, pensada para transformar las relaciones sociales con la naturaleza y el sistema político y económico, con la valorización de la esfera reproductiva defendida por la economía feminista.

Vega nos comparte la reflexión de la economista feminista Amaia Pérez Orozco (2005) que distingue entre las políticas de conciliación de la vida laboral y familiar, por un lado, y las políticas de igualdad de oportunidades en el mercado laboral, por otro lado, donde estas últimas no reconocen ni han reconocido la interrelación entre las esferas productivas y reproductivas. Por lo tanto, esta propuesta de políticas de igualdad de oportunidades no conversa con la categoría de sostenibilidad de la vida del Buen Vivir.

7 El término "indio permitido" fue cuñado por la socióloga aimara boliviana Silvia Rivera Cusicanqui de forma oral y usado por el antropólogo estadounidense Charles R. Hale en una publicación de 2004, pero sin mencionar cuándo fue usado originalmente por Cusicanqui. 
Es crucial dar centralidad a este tema del reconocimiento y valorización de la reproducción de la vida humana, ampliamente desarrollado por la economía feminista, pues se ha quedado ausente en las corrientes principales del pensamiento latinoamericano. Propuestas macroeconómicas como la Teoría de la Dependencia desarrollada por Enzo Faletto y Fernando Henrique Cardoso (1996) parecen no dejar la posibilidad de un análisis de género que permita entender la economía como una relación continua entre las esferas productivas y reproductivas, ficticiamente separadas por el liberalismo económico. Tampoco ha sido suficientemente analizado por los autores decoloniales; en palabras de la autora "Quijano, aun reconociendo el control del sexo y de la subjetividad como aspectos esenciales de la dominación/explotación, no los analiza" (Silvia VEGA, 2017, p. 48).

Marta Cabezas en su texto "Políticas de Género, Colonialidad y Neoliberalismo: Una Mirada Crítica al 'Proceso de Beijing' en Bolivia" hace una aguda crítica al movimiento feminista boliviano, que tacha de mestizo y blanco, dependiente de la financiación de las agencias de desarrollo extranjeras y al servicio de las entidades gubernamentales. Critica el proceso de preparación de la Conferencia de Beijing ${ }^{8}$ donde si bien se consultó a las mujeres indígenas, principalmente se colocó a las mujeres blancas y mestizas en el centro del debate y en la dirección del proceso. Las jerarquías establecidas en el proceso llevaron al entendimiento de la existencia de un ser mujer abstracto y universal. El proceso preparatorio para la Conferencia de Beijing se considera un hito en el movimiento feminista en todo el mundo, y es destacable la osadía de la autora al poner al descubierto los entramados racistas y clasistas que cubrieron ese proceso haciéndolo parecer como justo y revolucionario.

El texto de Cabezas nos pone especialmente de relieve el concepto de colonialismo interno de Pedro Gónzalez Casanova (2002), al mostrar como movimientos críticos a la modernidad, como el feminista, acaban siendo cooptados por las mujeres blancas (en Bolivia para el caso analizado, pero aplicable a los otros países de la región), quienes marcan una agenda que beneficia a las personas blancas de clase social media y alta, y mantienen sus intereses de continuar con un colonialismo interno, donde las mujeres indígenas y afrodescendientes están en posición de subordinación. La autora trae como esta situación cambia de rumbo con la llegada al poder del Movimiento al Socialismo (MAS), liderado por el indígena cocalero Evo Morales, que formula el Plan de lgualdad de Oportunidades 'Mujeres construyendo la nueva Bolivia para Vivir Bien' en 2008. La apertura del gobierno del MAS a la entrada y participación de las mujeres indígenas, que en 2017 representan la mitad del parlamento, colocó en jaque a las históricas ONG feministas del país; a partir de entonces, las agendas feministas serían marcadas por otras actoras. Beijing fue un proceso que desveló las diferencias entre las mujeres bolivianas que participaban: el nosotras y el ellas (ya que desde las Naciones Unidas se instó a la participación de las organizaciones de base en el Foro Alternativo de la Conferencia, pero en el caso boliviano se delegó a las mujeres blancas la organización de todo el proceso; por lo tanto, participaron mujeres blancas, mestizas e indígenas).

La autora también critica la práctica de gobiernos neoliberales, como en este caso del primer gobierno de Gonzalo Sánchez de Lozada entre 1993 y 1997, que integraron algunos sectores de la izquierda, entre ellos el movimiento feminista y de mujeres,

\footnotetext{
${ }^{8}$ El Proceso de Beijing se dio internacionalmente como preparación y articulación para la IV Conferencia de las Mujeres de las Naciones Unidas de 1995, comúnmente conocida como la Conferencia de Beijing, y se reconoce como una conquista histórica del movimiento de mujeres y feminista. El Foro Alternativo de ONG, un evento paralelo a la Conferencia contó con la presencia de más de 30.000 mujeres de todo el mundo, y tuvo resultados que marcaron el accionar del movimiento en las décadas siguientes.
} 
eminentemente blancos y de clase alta en aquella época, colocando el feminismo en función del neoliberalismo. Así, Cabezas afirma que "las ONGs han estado en el corazón de dos polémicas históricas del feminismo latinoamericano de la segunda ola. Me refiero al debate sobre su autonomía con respecto a los Estados y a la cooperación internacional del Norte, por una parte, y al debate sobre su carácter elitista y etnocéntrica, por otra" (Marta CABEZAS, 2017, p. 57).

En su texto "Género, pobreza y soberanía alimentaria", Xavier León analiza la situación de las mujeres rurales ecuatorianas, contrastándola con la búsqueda de la soberanía alimentaria del Buen Vivir. El machismo, la pobreza y, más recientemente, la creciente emigración masculina, que ha cambiado el paisaje rural del país, colocan a las mujeres rurales en una situación de gran vulnerabilidad. Esta situación, teniendo en cuenta que las mujeres son cada vez más las responsables de la agricultura familiar campesina, no contribuye a la efectividad del Buen Vivir, principalmente a la Soberanía Alimentaria que es uno de los derechos recogidos en la Constitución de 2008. Tras los datos arrojados en el texto de la relevancia numérica de las mujeres en el ámbito rural y su situación en desventaja con una feminización de la pobreza rural, el autor defiende que no se puede trabajar políticas públicas destinadas a alcanzar la Soberanía Alimentaria sin un adecuado enfoque de género.

Por su lado, Sarah A. Radcliffe analizará la interseccionalidad, donde se incluye el género, en las leyes, políticas públicas e instituciones ecuatorianas creadas para implementar el Buen Vivir, señalando los avances realizados y las limitaciones encontradas. Para la autora "la interseccionalidad no es la suma simple de las distintas discriminaciones" y "la categoría de 'género' [es] solo un comienzo de análisis y no un fin" (Sarah RADCLIFFE, 2017 , p. 75). En su texto "Género y Buen Vivir: Desigualdades Interseccionales y la Descolonización de las Jerarquías Persistentes", la autora da un especial énfasis en un análisis desde la geografía, resaltando las diferencias espaciales de la interseccionalidad en el Ecuador, que sigue una política pos-neoliberal centrada en los sistemas de redistribución y de protección social. La interseccionalidad se recoge en la Constitución ecuatoriana desde el principio de la diversidad y en las políticas públicas a través de las diferentes acciones afirmativas implementadas. El texto de Radcliffe es una llamada a descolonizarse, criticando conceptos usados desde las teorías de desarrollo, aunque la autora reconozca los beneficios que estas teorías han podido traer para trabajar con la interseccionalidad. Según la autora, conceptos como vulnerabilidad son eurocéntricos y deben descolonizarse (aunque la autora también use sin problematizar conceptos que fueron creados por las organizaciones de cooperación occidentales como bajo ingreso o discapacidad).

El texto de Sarah A. Radcliffe llama la atención por algunos errores ortográficos que confunden la lectura, así como por el uso de términos en inglés para esclarecer algunos conceptos usados en español, aspecto estético que nos hace preguntarnos cuál es el público meta a quien se dirige esta obra, pues ino sería más interesante usar términos en quichua o aimara que son las lenguas originarias de los países analizados? Por otro lado, el tema de género se diluye entre la interseccionalidad y deja de ser el centro de análisis en este artículo.

Margarita Manosalvas, en su texto "Políticas Públicas y Buen Vivir" analiza la verdadera presencia del concepto del Buen Vivir en las políticas públicas y lo hace a partir del reconocimiento del mandato constitucional que, en palabras de las compiladoras de la obra, rige "Ias políticas públicas son los medios a través de los cuales se deben hacer efectivos tales derechos" (VARELA; ZARAGOCín, 2017, p. 12). La autora establece una comparación entre los estados de bienestar en países europeos, considerados como resultado del crecimiento económico en un sistema capitalista, y las naciones de América 
Latina donde se han realizado políticas públicas dirigidas a poder alcanzar un crecimiento económico, crecimiento considerado necesario para llegar a tener un estado de bienestar, pero sin tener en cuenta las especificidades de la región. La autora se basa en el método de evaluación para la toma de decisiones políticas de Amartya Sen (2009), donde los derechos sociales pasan a ser integrantes de las políticas, como metas en forma de capacidades a ser alcanzadas. La dificultad que los gobiernos tienen en colocar los derechos sociales en política pública tiene raíces históricas que la autora ilustra para argumentar la indivisibilidad de todos los derechos humanos: no puede haber más jerarquía entre los derechos sociales y culturales, y los económicos y políticos. Llama la atención la ausencia de un análisis de género en este texto, si bien sí se propone una base para trabajar desde el género y desde otras formas de desigualdad.

Los dos últimos textos analizan como se ha trabajado la igualdad de género en la educación superior, poniendo de relieve los desafíos existentes. En "Ecuador: Igualdad de Género y Calidad Universitaria (2008-2013)", Erika Sylva Charvet argumenta con datos estadísticos como las brechas de género persisten en la universidad: a partir de prácticas machistas, de la falta de mujeres en cargos de dirección y de la concentración de alumnas en determinadas carreras universitarias que suelen llevar a posteriores ocupaciones laborales con salarios más bajos. La autora hace un interesante recorrido por la historia de la educación superior en el país, mostrando su carácter elitista y los procesos de privatización, carácter que se rompe con la actual Constitución que reconoce la educación superior como un bien público y un derecho humano; la última reforma universitaria de 2008 incorpora el Buen Vivir en el sistema educativo ecuatoriano, y la Ley Orgánica de Educación Superior de $\mathbf{2 0 1 0}$ ordena la formulación de políticas de acción afirmativa. Ahora bien, la autora muestra que, a pesar de los esfuerzos, plasmados principalmente a través de sistemas de cuotas y de participación, las desigualdades de género y raza se mantienen en las instituciones de educación superior en el período analizado de 2008-2012.

Paulina Quisaguano Mora critica en "Género, Inclusión y Educación Superior" el énfasis dado en las políticas educativas para fomentar el acceso de las personas más pobres, un énfasis realizado sin analizar los mecanismos de exclusión social históricamente construidos ni la diversidad social en su complejidad. La autora argumenta:

Actualmente, las luchas contra la injusticia requieren a la vez de reconocimiento y redistribución. La redistribución se refiere a la injusticia socioeconómica, arraigada en la estructura político-económica de la sociedad, pues incluye la explotación, la marginación económica, y la privación de los bienes materiales indispensables para llevar una vida digna. El reconocimiento se refiere a la injusticia cultural, esto implica que se tenga que hacer una re evaluación cada vez mayor de las identidades irrespetadas y de los productos culturales de grupos menospreciados. Tanto la injusticia socioeconómica como la injusticia cultural están arraigadas en procesos y prácticas que sistemáticamente ponen a unos grupos de personas en desventaja frente a otros. (Paulina QUISAGUANO MORA, 2017, p. 136).

La autora apuesta por un análisis interseccional que no sólo se valga de números de presencia de la diversidad entre el alumnado y el profesorado, sino que permee las prácticas educativas, los métodos y metodologías usados. Desde la teorización del feminismo negro y basándose en la feminista estadounidense bell hooks, la autora propone desarticular los sistemas patriarcales y racistas presentes en las instituciones educativas. Quisaguano Mora afirma que "Ia identificación de las obras de las mujeres de color y de clase obrera con lo 'experiencial' y los escritos de las mujeres blancas con la 'teoría' contribuye a reforzar ese racismo y elitismo" (QUISAGUANO MORA, 2017, p. 141), motivo por el cual bell hooks propone hacer teoría desde las mujeres negras. Esta diferenciación entre mujeres negras y mujeres blancas que la autora toma de bell hooks la podemos apreciar en el trabajo de Patricia Hill Collins (2002) cuando critica la asociación social que se hace de las mujeres negras con 
la sabiduría y la disociación de las mismas con el conocimiento. ${ }^{9}$ Este texto es una contribución muy interesante para el tema de esta obra, pues desde la sociedad civil se viene cuestionando que las realidades afroecuatorianas y afrocolombianas no están adecuadamente reflejadas en las propuestas del Buen Vivir, más centradas en las realidades indígenas y originarias. Lamentablemente este texto no se vincula con el concepto del Buen Vivir, presentándose como un escrito aislado de la obra, y perdiendo el potencial analítico y propositivo para mejorar las políticas y acciones en torno al Buen Vivir.

La epistemología, el método y la metodología cambian profundamente los resultados de las investigaciones, y en el caso de una ciencia comprometida y con objetivo de transformación social, cambian las políticas públicas y la implementación de las mismas. Este libro nos refleja exactamente eso, donde la elección de los objetos de investigación (pudiendo o no excluir a las mujeres y a las relaciones de género), de las ciencias y disciplinas a ser usadas de manera multidisciplinar o no (por ejemplo, al incluir la geografía encontramos especificidades de las mujeres rurales) y principalmente del sujeto de investigación (donde el ser mujer tiene otra relación con el objeto) y cuestionando ese sujeto de investigación (que históricamente presupone que las mujeres negras no producen conocimiento científico) ponen de relieve otras realidades y marcan otras acciones a ser realizadas en la búsqueda de la transformación social, en este caso caminando, o mejor hablando en femenino, tejiendo hacia el Buen Vivir.

Esta obra, en su búsqueda por aunar el feminismo y el Buen Vivir, nos dibuja utopías decoloniales, como bien indica el libro, basadas en análisis críticas de implementación de políticas públicas en Ecuador y Bolivia, y de la consolidación del movimiento de mujeres y feminista en Bolivia. Las críticas positivas y negativas, así como las propuestas apuntadas, abren las posibilidades para transformar esas utopías en acciones concretas, donde el Buen Vivir incorpore el Chacha Warmi en todo su accionar.

\section{Referencias}

ANDERSON, Benedict. Imagined communities: Reflections on the origin and spread of nationalism. Verso Books, New York / London: 2006.

CABEZAS, Marta. "Políticas de Género, Colonialidad y Neoliberalismo: Una Mirada Crítica al "Proceso a Beijing" en Bolivia". In.: VARELA, Soledad; ZARAGOCíN, Sofía (Compiladoras). Feminismo y Buen Vivir. Utopías decoloniales. Cuenca/Ecuador: PYDLOS Ediciones, 2017, p. 53-66.

CARDOSO, Fernando Henrique; FALETTO, Enzo. Dependencia y desarrollo en América Latina: ensayo de interpretación sociológica. México D.F.: Siglo XXI, 1996.

CHAVERT, Erika Sylva. "Ecuador: Igualdad de Género y Calidad Universitaria (2008-2013)". In.: VARELA, Soledad; ZARAGOCÍN, Sofía (Compiladoras). Feminismo y Buen Vivir. Utopías decoloniales. Cuenca/Ecuador: PYDLOS Ediciones, 2017, p. 103-133.

COLLINS, Patricia Hill. Black feminist thought: Knowledge, consciousness, and the politics of empowerment. New York / London: Routledge, 2002.

ESPINOSA DAMIÁN, Gisela. "Mujeres indígenas y derechos reproductivos. Fraguando modernidades alternativas". In: MILLÁN, Margarita. (Coordinadora). Más allá del feminismo: caminos para andar. México D. F.: Red de Feminismos Descoloniales, 2014. p. 247-275.

GALINDO, María. No se puede descolonizar sin despatriarcalizar: teoría y propuesta de la despatriarcalización. La Paz, Bolivia: Mujeres Creando, 2013.

\footnotetext{
${ }^{9}$ Traducción libre de la autora de la reseña: wisdom por sabiduría y knowledge por conocimiento, de la versión original de la obra Black Feminist Thought, de Patricia Hill Collins (2002).
}

8 Revista Estudos Feministas, Florianópolis, 26(3): e54775 
GONZÁLEZ CASANOVA, Pablo; ROITMAN, Marcos Roberto. Exploração, colonialismo e luta pela democracia na América Latina. Petrópolis: Vozes, 2002.

GONZÁLEZ GÓMEZ, Óscar. "Entre sodomitas y cuilonime, interpretaciones descoloniales sobre los 'indios vestidos de mujer' y la homosexualidad en los grupos nahuas del siglo XVI". In: MILLÁN, Margarita. (Coordinadora). Más allá del feminismo: caminos para andar. México D. F: Red de Feminismos Descoloniales, 2014. p. 277-298.

HALE, Charles R. "Rethinking Indigenous Politics in the Era of the 'Indio Permitido'". NACLA Report on the Americas, Report on Race, v. 38, n. 2, p. 16-21, 2004. Disponible en: https:/ /doi.org/10.1080/10714839.2004.11724509. Acceso en: 19/02/2018.

HERNÁNDEZ, Rosalva Aída. "Confrontando la Utopía Desarrollista: El Buen Vivir y La Comunalidad en las luchas de las Mujeres Indígenas". In.: VARELA, Soledad; ZARAGOCíN, Sofía (Compiladoras). Feminismo y Buen Vivir. Utopías decoloniales. Cuenca/Ecuador: PYDLOS Ediciones, 2017, p. 26-43.

LUGONES, María. "Colonialidad y género". Tabula rasa, n. 9, p. 73-101, jul.-dic. 2008. Disponible en: http://dev.revistatabularasa.org/numero-9/05lugones.pdf. Acceso en 19/ 02/1018.

PÉREZ OROZCO, Amaia. "Economía del género y economía feminista ¿Conciliación o ruptura?" Revista Venezolana de Estudios de la Mujer, v. 10, n. 24, p. 43-63, junio 2005.

QUIJANO, Aníbal. "Colonialidad del poder, eurocentrismo y América Latina." In: LANDER, Edgardo (Compilador). La colonialidad del saber: eurocentrismo y ciencias sociales. Perspectivas Latinoamericanas. Buenos Aires: CLACSO, Consejo Latinoamericano de Ciencias Sociales, 2000, p. 201-246.

QUISAGUANO MORA, Paulina. "Género, Inclusión y Educación Superior". In.: VARELA, Soledad; ZARAGOCÍN, Sofía (Compiladoras).Feminismo y Buen Vivir. Utopías decoloniales. Cuenca/ Ecuador: PYDLOS Ediciones, 2017, p. 134-143.

RADCLIFFE, Sarah. "Género y Buen Vivir: Desigualdades Interseccionales y la Descolonización de las Jerarquías Persistentes". In.: VARELA, Soledad; ZARAGOCíN, Sofía (Compiladoras). Feminismo y Buen Vivir. Utopías decoloniales. Cuenca/Ecuador: PYDLOS Ediciones, 2017, p. 75-90.

SEN, Amartya. The idea of justice. Massachusetts: The Belknap Press of Harvard University Press, 2009.

VARELA, Soledad; ZARAGOCíN, Sofía (Compiladoras). Feminismo y Buen Vivir. Utopías decoloniales. Cuenca/Ecuador: PYDLOS Ediciones, 2017. Disponible en: http:// dspace.ucuenca.edu.ec/handle/123456789/27831. Acceso en 11/02/2018.

VEGA, Silvia. "La Sostenibilidad de la vida como eje para Otro Mundo Posible". In.: VARELA, Soledad; ZARAGOCÍN, Sofía (Compiladoras). Feminismo y Buen Vivir. Utopías decoloniales. Cuenca/Ecuador: PYDLOS Ediciones, 2017, p. 44-52.

ZARAGOCÍN,Sofía. "Feminismo Decolonial y Buen Vivir". In.: VARELA, Soledad; ZARAGOCÍN Sofía (Compiladoras). Feminismo y Buen Vivir. Utopías decoloniales. Cuenca/Ecuador: PYDLOS Ediciones, 2017, p. 17-25.

[Recebida em 08/01/2018 e aceita em 19/03/2018]

Susana Martínez Martínez'

'Universidade de Brasília, Departamento de Línguas Estrangeiras e Tradução, Brasília, DF,

Brasil 
Susana Martínez Martínez (laresu@hotmail.com) é Professora Assistente Efetiva do curso Línguas Estrangeiras Aplicadas ao Multilinguismo e à Sociedade da Informação (LEA/ MSI) da Universidade de Brasília (UnB). Possui Mestrado em Gênero e Desenvolvimento pela Universidade Complutense de Madrid. Doutoranda em Estudos Comparados sobre as Américas no Departamento de Estudos Latino-americanos (ELA), na UnB. Pesquisadora do Laboratório de Estudos sobre Migrações Internacionais (LAEMI) e do MOBILANG, membro do Grupo de Estudos Interdisciplinares sobre Gênero (GRIEG) e Coordenadora do Projeto de Extensão Universitária Migrações e fronteiras no Distrito Federal: a integração linguística como garantia dos direitos humanos.

(ib) 0000-0001-7537-1883

10 Revista Estudos Feministas, Florianópolis, 26(3): e54775 